\title{
Towards robotics and artificial intelligence for the prevention of Covid 19 pandemic
}

\author{
Saloua SENHAJI, Sanaa FAQUIR, and Mohammed Ouazzani Jamil \\ Laboratoire Systèmes et Environnement Durables (SED), Faculté des sciences de l'ingénieur (FSI) \\ Université Privée de Fès ( UPF), Fez, Morocco
}

\begin{abstract}
In times of medical crisis, robotics and artificial intelligence helps humans manage emergencies and ensure a fast and efficient decontamination process. In this paper, we propose a robot with temperature detection, Corona virus checker using new biosensors, and artificial intelligence facial mask detection based on the deep convolutional neural network. Our robot can sterilize and patrol any type of area. In particular, airports, the train station and transport facilities which are the routes of transmission of the virus from one country to another.
\end{abstract}

\section{Introduction}

At the end of 2019, the first case of pneumonia of unknown origin was detected in Wuhan, China [1] High throughput sequencing revealed that this was a new severe acute respiratory syndrome $\beta$-coronavirus (SARS-CoV2) and a novel coronavirus disease (COVID-19) [2].

Through April 2020, the rapid spread of COVID-19 has impacted more than 200 countries with more than 900000 laboratory confirmed cases (with high numbers in China, United States, Spain, and Italy).

Global economic and social life has been seriously threatened since the World Health Organization (WHO) declared the pandemic $[3,4]$. Travel was particularly affected by the barriers used to maintain the social distance of disease management. Insufficient traditional measures used in these facilities exacerbated the medical crisis and helped COVID-19 to evolve from a national epidemiological stage into a terrifying international pandemic. How can the virus overwhelm the globe within months? If not, how does the severity of the disease differ from country to country?

The main factors are security measures! The Spanish flu killed more than 50 million people during the late 1920s. Fortunately, by applying our advanced technology today we can thwart such huge losses. 
Robotics and artificial intelligence have gained an increased presence to help manage the spread of COVID-19 in airports, hospitals [5], transportation and communities in general [6, 8]. Humanoid robots, autonomous vehicles, drones and other intelligent robots are used in many different ways to reduce human contact and the potential spread of the corona virus[13], including disinfecting and sterilizing public spaces, sensing or measuring body temperature, ensuring comfort to travelers. Although controversial in the past due to concerns about job losses and data privacy, the adoption of robotics and artificial intelligence in travel will likely continue after the COVID-19 pandemic becomes less severe [7].

For these various reasons, we believe it is urgent to conduct research on developing robots to combat the spread of the epidemic. In this paper, we present our smart robot Raspberry Pi with camera and biosensor that allows us to perform the following tasks:

Body temperature measurement function using a thermal sensors: The body temperature is measured for people in crowded places or high-risk areas of the epidemic. Through the analysis of infrared thermal map, the abnormal body temperature of people is judged and the alarm is given in time.

Optical biosensor for COVID-19: Jing Wang and all [9] has succeeded in developing a novel sensor to detect the new coronavirus. It could be used in the future to determine the concentration of viruses in the environment (The places where many people are present).

Detection of personnel wearing masks using the e LLE-CNNs and a voice reminder for those who do not wear masks.

\section{Robot types and applications}

While robots have played a fundamental role in industrial environments and high-risk jobs for many years, little attention has been paid to the roles that robots can play in "highly connected" or "relational" environments, High interpersonal skills, "such as transportation. Researchers use the word" robotics "to describe the research field of information engineering, computer science, and other technical fields [10] where robots are defined as" a programmable operational mechanism on two or more axes " With a certain degree of independence, moving in its environment, to perform the tasks as expected "[11, 12].

Researchers have gradually come to classify human-robot interaction (HRI) into three categories: robot-centered approaches, human-centered approaches, and robot cognitioncenter approaches [17]. Based on the nature of the application,[18] further classified robots interacting with humans into "telerobot," "teleoperator," and "social robot" (Table 1).

Table 1. The classification of robots interact with humans.

\begin{tabular}{|c|c|c|c|}
\hline Classification Name & Definition & Function & Types of Robots \\
\hline Telerobot & $\begin{array}{l}\text { Robots that can sense } \\
\text { the environment and } \\
\text { make limited } \\
\text { automatic reactions } \\
\text { through computer } \\
\text { programs to complete } \\
\text { routine tasks }\end{array}$ & Routine tasks & $\begin{array}{l}\text { Picking and placing } \\
\text { robots, welding } \\
\text { robots, cleaning } \\
\text { robots, delivery } \\
\text { robots, self-guard } \\
\text { gate, ultraviolet-light- } \\
\text { disinfection robots }\end{array}$ \\
\hline Teleoperator & $\begin{array}{l}\text { Robots that deal with } \\
\text { nonroutine tasks in } \\
\text { hazardous or } \\
\text { inaccessible }\end{array}$ & Nonroutine tasks & $\begin{array}{l}\text { Drones, unmanned } \\
\text { spacecraft, undersea } \\
\text { robotic vehicles, }\end{array}$ \\
\hline
\end{tabular}




\begin{tabular}{|c|c|c|c|}
\hline & $\begin{array}{c}\text { environments with } \\
\text { continuous remote } \\
\text { control from humans. }\end{array}$ & & $\begin{array}{c}\text { unscrewed aerial } \\
\text { vehicles }\end{array}$ \\
\hline Social robot & $\begin{array}{c}\text { Robots that have } \\
\text { autonomous agents } \\
\text { with social } \\
\text { intelligence to } \\
\text { interact with humans } \\
\text { in an acceptable } \\
\text { manner. }\end{array}$ & $\begin{array}{c}\text { Entertainment, } \\
\text { teaching, comfort, and } \\
\text { assistance }\end{array}$ & $\begin{array}{c}\text { Guiding robots, } \\
\text { teaching robots, } \\
\text { communication } \\
\text { robots, assistive } \\
\text { healthcare robots, } \\
\text { autonomous vehicles }\end{array}$ \\
& & & \\
\hline
\end{tabular}

With the development of artificial intelligence (AI), robots became equipped with "social intelligence" - the ability to replace human [13]. Contrary to industrial robots widely used in agriculture, manufacturing, and medicine [15], a smart robot socially emphasizes interacting with humans through its use of artificial intelligence [16].

\section{Robotics and artificial intelligence for the prevention of Covid- 19 pandemic in airports}

Most major airports manage a huge flow of people, many of whom require information, navigation and pick-up transport services. Prior to the COVID-19 pandemic, some airports had begun to pilot the use of robots to assist airport managers in providing airport security, providing directional information and services, assisting with passenger check-in, delivering luggage and certain other routine services.

As the COVID-19 pandemic placed greater value on public health, the opportunities and confidence of airport robots have increased. Robots for disinfection, sterilization, detection of masks, and which measures the temperature of travelers have now acquired a secure place in many airports around the world.

In this paper, we propose a robot to help the authorities for the prevention of virus. Our robot is equipped with a temperature detection, Corona virus checker using a biosensors [9], and artificial intelligence facial mask detection based on the deep convolutional neural network.

\subsection{Body temperature measurement function:}

Measuring the outside temperature has advanced the technology while adding detection techniques, ensuring respect for the distance to the patient, improving accuracy and creating better monitoring tools. Its data can be interesting for patients with corona virus. We use digital temperature sensors to support the wide range of accuracy, and performance conditions amongst the different applications.

We can choose from a variety of sensors to translate temperature phenomena into a measurable signal. Three common sensor varieties are the infrared thermometer, the thermal camera or infrared camera. Each has its own operating principles, benefits, considerations, and drawbacks. 
All three use the principle of infrared radiation. Natural light is emitted both in visible light but also in other "radiations" including ultraviolet and infrared. The artificial light sources can, depending on the model, emit in the visible, infrared or ultraviolet. All bodies (inert objects and living beings) "reflect" all this radiation.

Our eye is only able to see visible light, some devices can see in other radiation. Infrared is the type of radiation that best highlights overheating, which is why thermometers without contact and thermal imaging cameras use this radiation.

In this paper, we use a thermal camera. The specific thermal camera for the human body can be placed at a higher distance to target, depending on the model from 1 meter. Unlike the thermometer, we can see on the camera screen an area wider. Since a maximum temperature detection function is present, the camera will be able to systematically measure the hottest point of the head of the person to be tested (fig 1). This allows you to make less mistakes compared to an infrared thermometer, more so than a camera thermal has very good thermal sensitivity (often better than $0.05-0.07^{\circ} \mathrm{C}$ ).

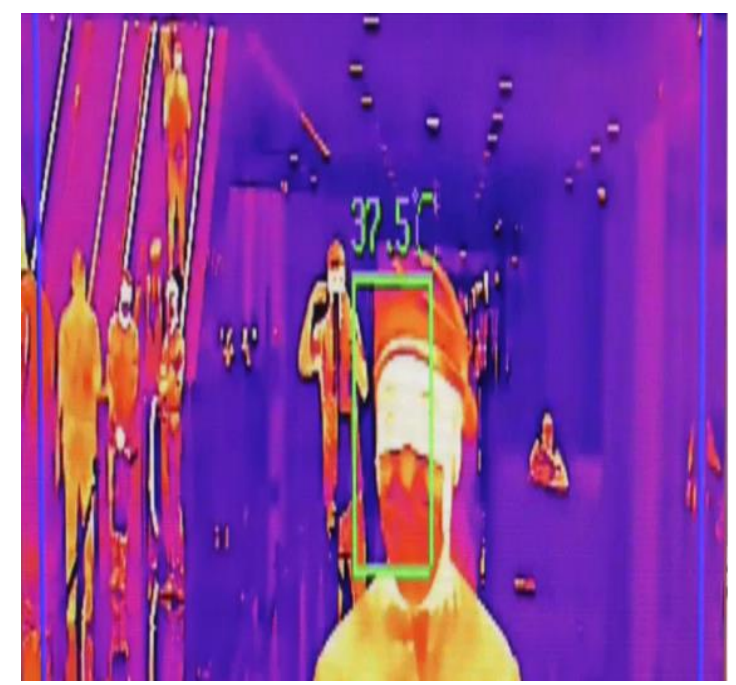

Fig 1: Detecting highest temperature with thermal camera

\subsection{Detection of personnel wearing masks :}

Face masks have become a part of daily lives in the age of the coronavirus. To prevent people from acquiring the coronavirus, respiratory or infectious pathogen, and blocking larger particles from sneezes or coughs of asymptomatic people, face-covering with surgical or face masks is mandatory in many places such as office buildings, hospitals, public transportation facilities or even retail stores and restaurants.

in the present paper we use an advanced LLE-CNNs -based facial recognition solution[19] with mask detection can quickly identify and track everyone in a crowd as they move, and simultaneously help recognize unmasked people and restrict access. LLE-CNNs for masked face detection, which consist of three major modules. The Proposal module first combines two pre-trained CNNs to extract candidate facial regions from the input image and represent them with high dimensional descriptors.

After that, the embedding module is incorporated to turn such descriptors into a similaritybased descriptor by using locally linear embedding (LLE) algorithm and the dictionaries trained on a large pool of synthesized normal faces, masked faces and non-faces. 
For the test, we introduces a dataset, denoted as MAFA (fig 2, fig 3, fig 4), with 30811 Internet images and 35806 masked faces. Faces in the dataset have various orientations and occlusion degrees, while at least one part of each face is occluded by mask.
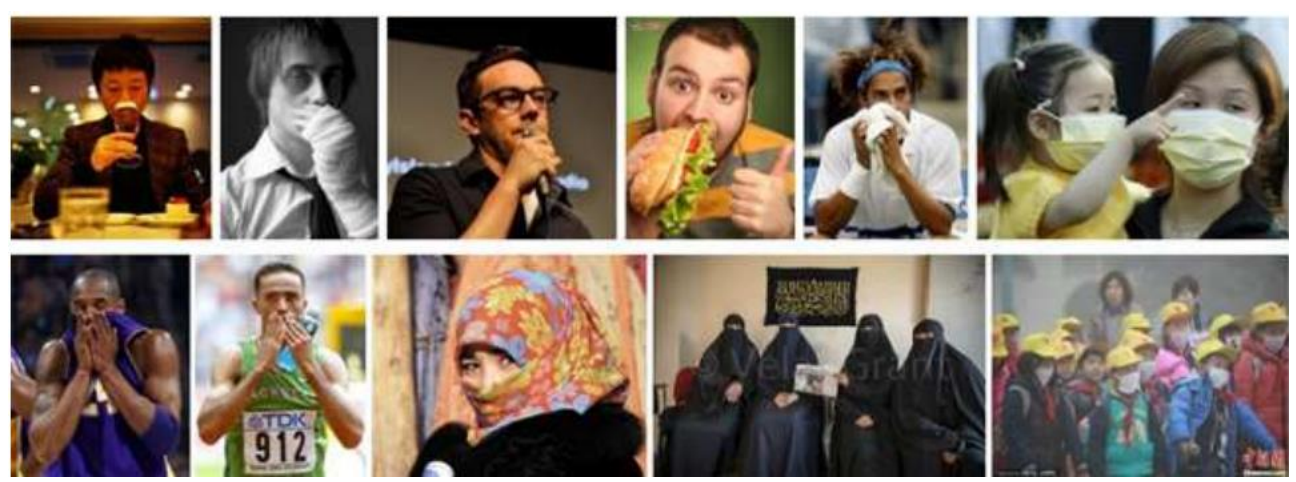

Fig 2: Masked faces may have diversified orientations, degrees of occlusion and mask types, making their detection an extremely challenging task for existing face detectors.

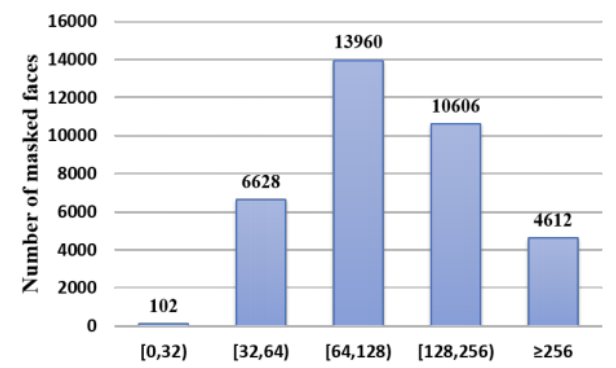

(a) Face Size

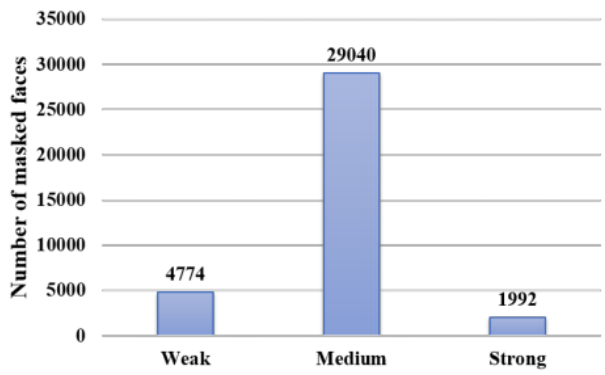

(c) Occlusion Degree

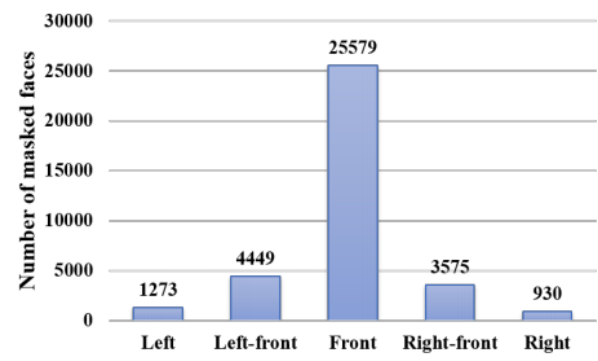

(b) Face Orientation

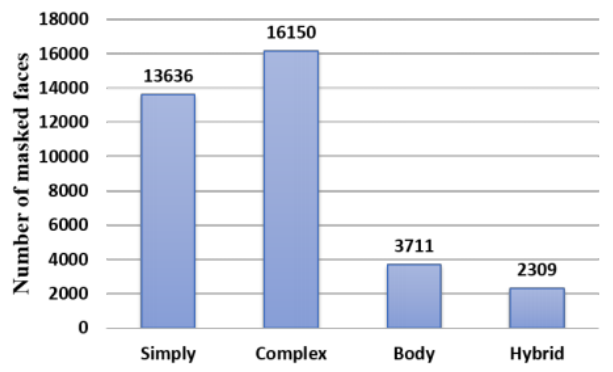

(d) Mask Type

Fig 3 : Statistics of the masked faces in MAFA. 


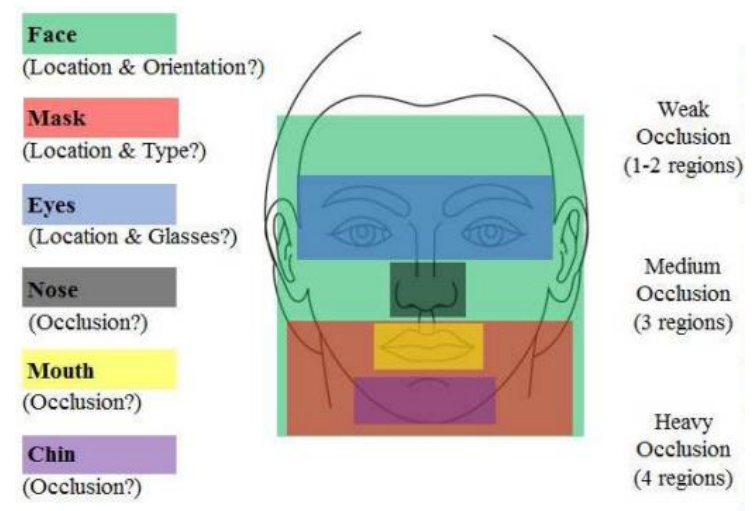

Fig 4: Definition of facial attributes for annotation

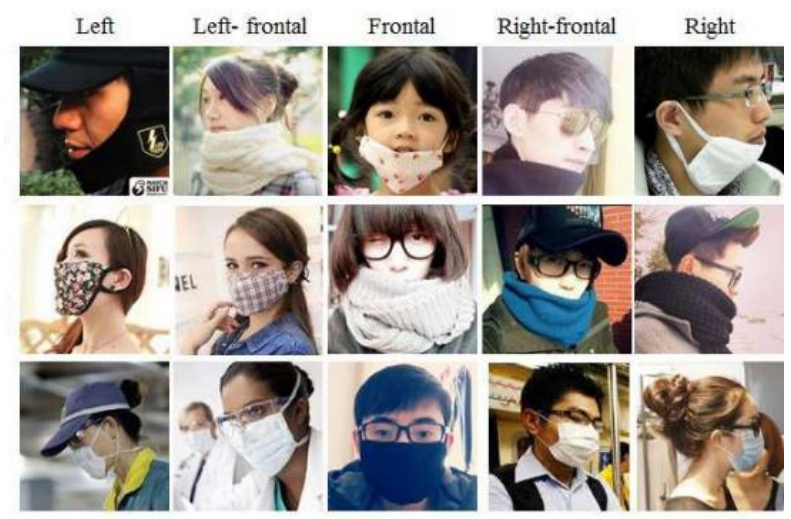

Fig 5: Representative facial image with different attributes

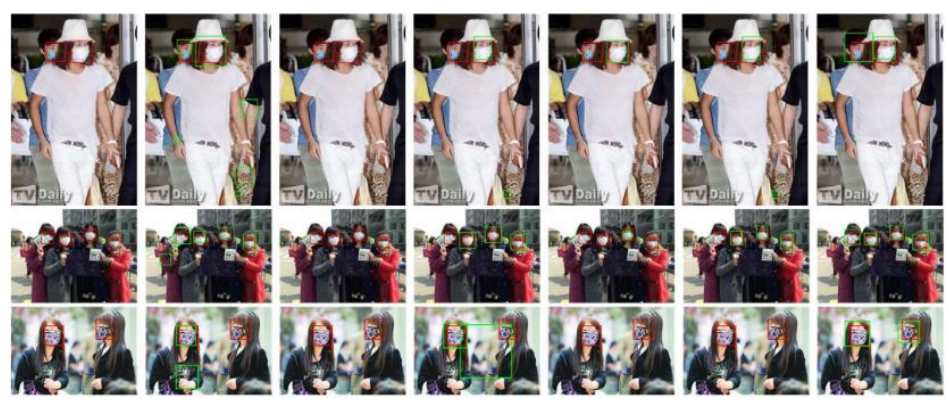

Fig 6. Representative detection results of the approach used

\subsection{Intelligent analysis:}

Fast information collection and real-time processing. The returned information is automatically processed by the system, which is convenient for the robot to make a quick decision. 


\subsection{Corona virus test using new optical biosensor :}

Jing Wang and all [9] have developed an alternative test method, in the form of an optical biosensor. The sensor combines two different effects to detect the virus: one optical and one thermal. The sensor is based on tiny structures of gold, on a glass substrate. The nanoislands are home to artificially produced DNA sequences that match specific RNA sequences of the SARS-CoV-2 virus. The new coronavirus is a so-called RNA virus: its genome consists of a single RNA strand. The artificial DNA receptors on the sensor are thus the complementary sequences to the unique RNA genome sequences of the virus, which can clearly identify it. The technology used by researchers to detect the virus is LSPR. This is an optical phenomenon that occurs in metallic nanostructures. When molecules dock on the surface, the optical refractive index changes in this plasmonic near field. With an optical sensor located on the back of the sensor, this can be measured and thus determined whether the desired RNA strands are in the sample.

The images collected by the inspection robot can be monitored in real time, and the information such as on-site video, infrared temperature measurement image of human body and whether to wear masks can be sent back to the main control room through wireless network in real time.

\section{Conclusion}

In this paper, we have presented a robot with temperature detection, Corona virus checker using new biosensors, and artificial intelligence facial mask detection based on the deep convolutional neural network CNN, the adoption of our robots in airport can Reducing human contact and making use of autonomous unmanned machines to carry out a risky task, Reducing labor consumption and number of worker on boats and plane boards, effectively reducing the risk of infection, On-spot temperature checkups which helps medical staff monitoring people in airports and waiting rooms and leaves no room for human error.

\section{References}

1. Huang, C.; Wang, Y.; Li, X.; Ren, L.; Zhao, J.; Hu, Y.; Zhang, L.; Fan, G.; Xu, J.; Gu, X. Clinical Features of Patients Infected with 2019 Novel Coronavirus in Wuhan, China. Lancet 2020, 395, 497-506. (2)

2. Lu, R.; Zhao, X.; Li, J.; Niu, P.; Yang, B.; Wu, H.; Wang, W.; Song, H.; Huang, B.; Zhu, N. Genomic Characterisation and Epidemiology of 2019 Novel Coronavirus: Implications for Virus Origins and Receptor Binding. Lancet 2020, 395, 565-574.

3. Zhang, M. Litvnova, W. Wang, Y. Wang, X. Deng, X. Chen, M. Li, W. Zheng, L. Yi, X. Chen, Q. Wu, Y. Liang, X. Wang, J. Yang, K. Sun, I.M. Longini Jr., M.E. Halloran, P. Wu, B.J. Cowli ng, S. Merler, C. Viboud, A. Vespignani, M. Ajelli, H. YuEvolving epidemiology and transmission dynamics of coronavirus disease 2019 outside Hubei province, China: a descriptive and modelling study . Lancet Infect Dis. (2020), 10.1016/S1473-3099(20)30230-9

4. Huang, Y. Wang, X. Li, L. Ren, J. Zhao, Y. Hu, L. Zhang, G. Fan, J. Xu, X. Gu, Z. Cheng, T. Y u, J. Xia, Y. Wei, W. Wu, X. Xie, W. Yin, H. Li, M. Liu, Y. Xiao, H. Gao, L. Guo, J. Xie, G. Wa ng, R. Jiang, Z. Gao, Q. Jin, J. Wang, B. CaoClinical features of patients infected with 2019 novel coronavirus in Wuhan, China Lancet, 395 (2020), pp. 497-506. 
5. Bowen, J., \& Morosan, C. (2018). Beware hospitality industry: The robots are coming. Worldwide Hospitality and Tourism Themes, 10(6), 726-733.

6. Bhimasta, R. A., \& Kuo, P. Y. (2019, September). What causes the adoption failure of service robots? A case of Henn-na Hotel in Japan [Paper presentation]. Adjunct Proceedings of the 2019 ACM International Joint Conference on Pervasive and Ubiquitous Computing and Proceedings of the 2019 ACM International Symposium on Wearable Computers, London, UK.

7. Buheji, M., \& Ahmed, D. (2020). Foresight of Coronavirus (COVID-19) opportunities for a better world. American Journal of Economics, 10(2), 97-108.

8. Cain Lisa, N., Thomas John, H., \& Alonso, M. Jr, (2019). From sci-fi to sci-fact: The state of robotics and $\mathrm{AI}$ in the hospitality industry. Journal of Hospitality and Tourism Technology, 10(4), 624-650.

9. Jing Wang \& all, Optical biosensor for COVID-19. (2020) : being published

10. McKerrow, P. J., \& McKerrow, P. (1991). Introduction to robotics. Addison-Wesley Sydney.

11. Ivanov, S., \& Webster, C. (2019a). Conceptual Framework of the Use of Robots, Artificial Intelligence and Service Automation in Travel, Tourism, and Hospitality Companies.

In I. Stanislav \& W. Craig (Eds.), Robots, Artificial Intelligence, and Service Automation in Travel, Tourism and Hospitality (pp. 7-37).

12. Ivanov, S., \& Webster, C. (2019b). Robots, artificial ilntelligence, and service automation in travel, tourism and hospitality.

13. Lazzeri, N., Mazzei, D., Zaraki, A., \& De Rossi, D. (2013, July). Towards a believable social robot [Paper presentation]. Conference on Biomimetic and Biohybrid Systems, London, UK.

14. Marr, B. (2020, March 18). Robots and drones are now used to fight COVID-19.

15. Engelberger, J. F. (2012). Robotics in practice: Management and applications of industrial robots. Springer Science \& Business Media.

16. Dautenhahn, K., Billard, A. (1999, April). Bringing up robots or-the psychology of socially intelligent robots: From theory to implementation. In Proceedings of the Third Annual Conference on Autonomous Agents (pp. 366-367).

17. Dautenhahn, K. (2007). Socially intelligent robots: Dimensions of human-robot interaction. Philosophical Transactions of the Royal Society B: Biological Sciences, 362(1480), 679-704.

18. Sheridan, T. B. (2016). Human-Robot interaction: Status and challenges. Human Factors: The Journal of the Human Factors and Ergonomics Society, 58(4), 525-532.

19. K. Zhang, Z. Zhang, Z. Li, and Y. Qiao. Joint face detection and alignment using multitask cascaded convolutional networks. IEEE Signal Processing Letters, 23(10):1499-1503, 2016. 